\title{
Influencia de las aletas sobre el tiempo de ejecución en los rescates de víctimas con parada cardiorespiratoria \\ Influence of fins on the lifeguard answer's time to rescue victims with a cardiorespiratory arrest Ismael Sanz Arribas, RaquelAguado Gómez y Vicente Martínez de Haro \\ Universidad Autónoma de Madrid (España)
}

Resumen. El objetivo de este estudio es conocer la influencia del empleo de las aletas, sobre el tiempo que los socorristas tardan en rescatar a una víctima con parada cardiorespiratoira del agua. Reducir el tiempo de este eslabón de la cadena de supervivencia, es fundamental para que se puedan iniciar lo antes posible las maniobras de Reanimación Cardiopulmonar (RCP). 45 aspirantes a socorrista de ambos sexos han realizado dos test de 50 metros de rescate a una víctima inconsciente ( 25 metros de nado de aproximación y 25 metros de arrastre de la víctima). El test 1 se hace sin aletas y el test 2 se hace con aletas. En el test 2, los socorristas inician la prueba sin tener las aletas colocadas. Los resultados de este estudio demuestran que en general, las aletas son adecuadas para todos los socorristas estudiados, pero son especialmente recomendables para aquellos socorristas, cuyo nivel de destreza en el agua es más bajo. Así, los sujetos que obtuvieron peores marcas en el test 1 (sin aletas), consiguen mejorar los resultados en el test 2 (con aletas) (p $<0,001$ ). En cambio, los participantes que obtuvieron mejores marcas en el test 1 (sin aletas), no mejoraron significativamente el tiempo en el test 2 (con aletas) (p=0,896). En definitiva, se puede afirmar que las aletas mejoran las condiciones en las que el socorrista inicia las maniobras de RCP, ayudan a incrementar las probabilidades de supervivencia de las víctimas con parada cardiorespiratoria y mejoran la seguridad de los socorristas durante el rescate. Palabras clave. Salvamento, aletas, ahogamientos, socorrista, reanimación cardiopulmonar.

Abstract. The aim of this study is to know how the use of fins influence on the lifeguard answer's time to rescue victims with a cardiorespiratory arrest. It's essential to star as soon as possible the Cardiopulmonary Resuscitation (CPR) procedure to reduce that answer's time. 45 candidates to lifeguard, of both sexs, have carried out 2 different rescue tests of unconscious victim to a distance of 50 meters (25 swimming approach meters and 25 meters carrying the victim). Test number 1 has been done without fins and test number 2 with fins. On test number 2, lifeguards started without their fins. The results of this study prove that as a rule the use of fins are suitable for all the lifeguards studied, but mainly for those lifeguards whose level is lower. So, those who obtain worse scores on test number 1 ( no fins ) achieve better scores on test number 2 ( fins ) ( $p<0,001$ ) and those who obtain better scores on test number 1 don't improve considerably their times on test number $2(p=0,896)$. To sum up, we can say that the use of fins will improve the conditions in which the CPR procedure will be started by the lifeguard, helping to increase the survival options of the victims with a cardiorespiratory arrest and improving lifeguards security during the rescue.

Keywords. rescue, fins, lifeguard, drowning, cardiopulmonary resuscitation.

\section{Introducción}

Puesto que en la mayoría de los casos no es viable iniciar las maniobras de RCP dentro del agua, es fundamental trasladar lo antes posible a los ahogados con parada cardiorespiratoria hasta la orilla y de ese modo, iniciar la RCP con opciones de éxito. Por lo tanto, el objetivo de este estudio es conocer la influencia del empleo de las aletas, sobre el tiempo de rescate a una víctima con parada cardiorespiratoria.

La «cadena de supervivencia de ahogamientos» consta de los siguientes pasos: prevenir el ahogamiento, reconocer un ahogamiento, llevar a la superficie al ahogado, extraerle del agua y proporcionar atención médica, incluyendo RCP si es necesario (1). Por este motivo, es imprescindible reducir el tiempo de todos los eslabones de la cadena de salvamento para lograr la atención médica temprana.

En noviembre de 2014, la Organización Mundial de la Salud (2) informaba que alrededor de 370.000 personas pierden la vida cada año como consecuencia de ahogamientos en medios acuáticos. Siendo el ahogamiento una de las principales causas de muerte accidental de niños y jóvenes en todo el mundo. Según la Federación Española de Salvamento y Socorrismo, los espacios acuáticos españoles fueron el escenario de la muerte por ahogamiento de 419 personas en el año 2015, de las cuales, 240 se produjeron entre los meses de junio y septiembre $(3,4)$.

A pesar de estas cifras tan dramáticas, muchos estudios demuestran que la calidad y cantidad de recursos que se destinan a la seguridad de los bañistas, es mejorable (5-12). En este sentido, también se sabe que las instalaciones que no tienen servicio de salvamento, son mucho más peligrosas que las que lo tienen $(3,4,10)$.

Es muy importante suministrar a los socorristas el material auxiliar de rescate apropiado, ya que las posibilidades de tener éxito en los rescates acuáticos, son mayores cuando el socorrista dispone de material auxiliar de salvamento (12-15). La lista de materiales auxiliares de salvamento es muy extensa y la mayoría de ellos son enormemente

Fecha recepción: 15-06-16. Fecha de aceptación: 17-10-16

Vicente Martínez de Haro

vicente.martinez@uam.es útiles en los rescates acuáticos. Algunos autores afirman que uno de los materiales de rescate más ventajosos son las aletas, ya que ayudan a mantener al socorrista y a la víctima sobre el agua, mejoran la seguridad, disminuye el tiempo de intervención de un rescate acuático en playas y en piscinas, en distancias largas y cortas, aunque las aletas son más ventajosas en las distancias largas (12, 13, 15-20). La disminución del tiempo de rescate, es un factor clave para las víctimas que están en parada cardiorespiratoria, ya que cuanto más tiempo transcurre sin asistir a este tipo de víctimas, menores son sus posibilidades de supervivencia (21-23). Las aletas también pueden ayudar a disminuir la fatiga de los socorristas en los rescates, y está demostrado que si el socorrista está menos cansado durante la reanimación cardiopulmonar, la maniobra será más efectiva (24). Por otro lado, las olas y corrientes son la causa de muchos ahogamientos y además, aumentan el riesgo y la dificultad de los socorristas en las intervenciones (25). Además, hay investigaciones que demuestran que cuando los socorristas intervienen con ayuda de las aletas en playas, no sólo tardan menos tiempo, sino que además, recorren menos distancia que los que no utilizan aletas (16). En relación al tipo de aletas, los estudios señalan que cualquier tipo de aleta es mejor que no llevar ninguna $(13,17)$. Aunque se podría afirmar que en general, las aletas más rígidas y largas son más ventajosas que las aletas blandas y cortas (16-18). Por si esto fuera poco, las aletas son un material muy asequible y duradero.

Como puede observarse, existen bastantes estudios que señalan que la utilización de aletas, incrementa las posibilidades de éxito en los rescates acuáticos, incluso, algunos autores señalan que el tiempo de rescate mejora sobre todo en los socorristas con peor nivel de destreza (20), pero no se han encontrado datos cuantitativos que acompañen esta última afirmación. Por lo tanto, en este trabajo se medirán por un lado, los beneficios del empleo de las aletas y por otro, se cuantificarán los beneficios de la utilización de las aletas, dependiendo del nivel de destreza del socorrista.

\section{Material y métodos}

\section{Descripción de la muestra}

La población que ha participado en este estudio está formada por 
45 aspirantes a socorrista acuático de ambos sexos, que cursaron y aprobaron el curso de socorrista de una entidad, cuya sede social se encuentra en la Comunidad Autónoma de Madrid (España). Por lo tanto, cumplen los requisitos mínimos legales para ser socorrista, es decir: Son mayores de 16 años, han superado el $4^{\circ}$ curso de la Enseñanza Secundaria Obligatoria (E.S.O.) o equivalente. Además han presentado un certificado médico oficial, en el que consta que no padecen enfermedad infecto-contagiosa y que son aptos para realizar ejercicio físico en el agua.

\section{Material e instalaciones}

El material que se ha empleado para la realización de este estudio es el siguiente:

- Un cronómetro modelo Casio® HS-30W, resistente al agua y con memoria para 10 lap/Split.

- Un maniquí de arrastre para el agua, homologado por la Federación Española de Salvamento y Socorrismo.

- Aletas de la marca Cressi® , modelo «Clio», utilizadas habitualmente para la natación y el buceo recreativo.

- Un silbato.

- Un colaborador para sujetar el maniquí en posición vertical cerca de la superficie del agua.

- Una calle de 25 metros de largo y 2 metros de ancho, ubicada en un vaso de piscina climatizada.

\section{Metodología}

Para cuantificar los beneficios de las aletas sobre la población de estudio y sobre los diferentes niveles de destreza de la muestra, se ha diseñado una prueba que será realizada en dos ocasiones por todos los participantes del estudio (test 1 y test 2). En primer lugar, toda la muestra realizará la prueba sin aletas (test 1) y después de recuperar completamente la fatiga, repetirán la misma prueba, pero con ayuda de aletas (test 2). Los resultados del test 1 (sin aletas) serán organizados en tres grupos de 15 sujetos cada uno, pero con un nivel de destreza estadísticamente diferente (grupo 1 de nivel alto, grupo 2 de nivel medio y grupo 3 de nivel bajo). Después de realizar el test con aletas (test 2), se cuantificarán los posibles beneficios del total de la muestra y también, de cada uno de los grupos con distinto nivel de destreza que se formaron en el test 1 (sin aletas).

\section{Descripción de la prueba}

La prueba diseñada para este estudio simula el rescate acuático de una víctima inconsciente, que se ahoga en un espacio acuático de aguas tranquilas. La muestra realizó la prueba en dos ocasiones «test 1 » y «test 2». En el test 1, se realizó la prueba sin aletas y en el test 2 se realizó la prueba con aletas, incluyendo el tiempo de colocación del material.

Toda la muestra ha sido entrenada previamente en las técnicas necesarias para el desarrollo de ambos test.

Descripción de la prueba sin aletas (test 1):

- El sujeto se coloca de pie sobre el bordillo del vaso.

- Tras el pitido de salida, se zambulle en el agua con la técnica de paso de gigante y manteniendo la cabeza fuera del agua.

- Realiza 25 metros de nado de aproximación a estilo de crol con la cabeza por fuera del agua.

- Después de contactar con el maniquí, el participante debe colocarlo boca arriba para iniciar el remolque.

- Hasta que el aspirante llega al maniquí, este será agarrado por un colaborador en las siguientes condiciones:

- En posición vertical y completamente lleno de agua.

- La cara del maniquí está bajo el agua y orientada hacia el rescatador. La parte trasera de la cabeza, está tocando la pared del bordillo opuesto al de salida del participante.

- El colaborador sujeta el maniquí desde fuera del agua.

- Los 25 metros de remolque del maniquí, se realizan con una técnica adecuada para víctimas inconscientes. Por lo tanto, la cara y las vías respiratorias del maniquí, deben permanecer por encima del agua en todo momento. Se recomienda a los socorristas emplear la patada de braza dorsal $(16,26)$.

- El final de la prueba se produce cuando el aspirante a socorrista, toca la pared del bordillo desde el que partió.

Descripción del «test 2» (con aletas):

- El «test 2» es exactamente igual que el test 1 (sin aletas), pero el participante inicia la prueba en pie sobre el bordillo y con las aletas en el suelo. Después del pitido de salida, el sujeto recoge del suelo las aletas, se zambulle en el agua con ellas y sin hundir la cabeza. Una vez dentro del agua se coloca las aletas. El resto del «test 2» se desarrolla del mismo modo que el «test $1 »$.

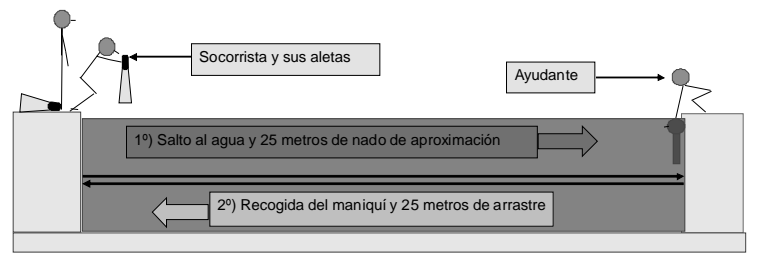

Figura 1. Representación gráfica de la prueba.

Para comprobar si el empleo de las aletas (test 2), proporciona mayores beneficios a aquellos aspirantes a socorristas que disponen de un menor nivel de destreza en el agua, se ha dividido toda la muestra en tres grupos de tres niveles diferentes, dependiendo del resultado obtenido en test 1 (sin aletas). Para hacer la división, se han determinado los percentiles 33 y 66 de la muestra total, formándose tres grupos de 15 participantes cada uno, con tres niveles de destreza diferentes entre sí:

- Grupo A: 15 participantes que emplearon menos de 70" en el test 1 (sin aletas).

- Grupo B: 15 participantes, que emplearon más de 70" y menos de 87" en el test 1 (sin aletas).

- Grupo C: 15 participantes que emplearon más de 87" en el test 1 (sin aletas).

Después de realizar el test 2 (con aletas) se ha vuelto a agrupar la muestra, tomando como referencia los rangos de tiempo que delimitaron los tres niveles de destreza en el test 1 (sin aletas). Cuando se ha aplicado este mismo criterio de agrupación sobre las marcas obtenidas en el test 2 (con aletas), se han formado sólo dos grupos, ya que ningún aspirante empleó más de 87" en realizar el test 2 (con aletas):

- Grupo A con aletas: Formado por 35 participantes, que emplearon menos de 70" en el test 2.

- Grupo B con aletas: Formado por 10 participantes que emplearon más de 70" en el test 2 .

\section{Resultados}

\section{Estadísticos descriptivos}

Tabla 1

Estadísticos descriptivos de los tiempos del test 1 (sin aletas) y el test 2 (con aletas

\begin{tabular}{cccc}
\hline & \multicolumn{2}{c}{ Estadísticos } & \\
\hline & Sin aletas & Con aletas & $\begin{array}{c}\text { Diferencia de tiempo } \\
\text { entre test 1 y test 2 }\end{array}$ \\
\hline N & 45 & 45 & 45 \\
\hline Media en segundos & 83,20 & 64,31 & 18,87 \\
\hline Mediana en segundos & 77,00 & 62,00 & 12,00 \\
\hline Moda en segundos & 71 & 56 & 0 \\
\hline Desv. tip. En segundos & 25,34 & 9,57 & 23,96 \\
\hline
\end{tabular}

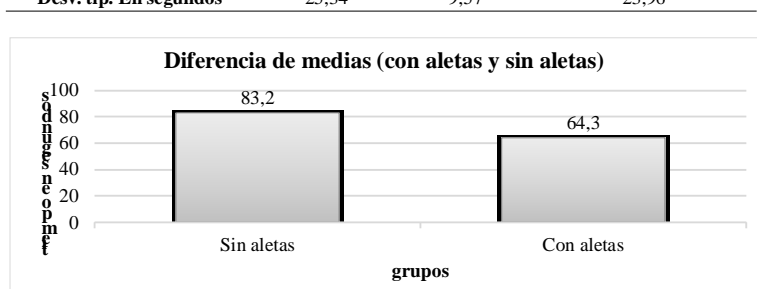

Figura 2, Diferencia de medias entre el test 1 (sin aletas) y el test 2 (con aletas)

La desviación típica de la muestra en la prueba sin aletas es 25,34 segundos y en la prueba con aletas es 9,57 segundos. 


\section{Prueba de normalidad}

La prueba de normalidad Shapiro-Wilk determina que las muestras no son normales $(\mathrm{p}<0,001)$ en el test 1 sin aletas y $(\mathrm{p}<0,007)$ en el test 2 con aletas.

Prueba de los rangos con signo de Wilcoxon

La prueba de rangos con signo de Wilcoxon diferencia entre el test 1 (sin aletas) y el test 2 (con aletas) indicando una mejora significativa $(\mathrm{p}<0,001)$

\section{Descriptivos de los grupos de aspirantes sin aletas y con aletas:}

Tabla 2

Estadísticos descriptivos de los tiempos del test 1 (sin aletas) y el test 2 (con aletas) de los grupos formado

\begin{tabular}{|c|c|c|c|c|c|}
\hline & $\begin{array}{c}\text { Grupo A sin } \\
\text { aletas } \\
(<70 ")\end{array}$ & $\begin{array}{c}\text { Grupo B sin } \\
\text { aletas } \\
(>70 " y<87 ")\end{array}$ & $\begin{array}{c}\text { Grupo C } \\
\text { sin aletas } \\
(>87 ")\end{array}$ & $\begin{array}{c}\text { Grupo A } \\
\text { con aletas } \\
\text { (<70") }\end{array}$ & $\begin{array}{c}\text { Grupo B con } \\
\text { aletas } \\
(>70 " y<87 ")\end{array}$ \\
\hline $\mathbf{N}$ & 15 & 15 & 15 & 35 & 10 \\
\hline Media en segundos & 62,73 & 76,60 & 110,27 & 60,09 & 79,10 \\
\hline Moda en segundos & 70 & 71 & 94 & 56 & 78 \\
\hline Desv. típ. En segundos & 5,3 & 4,7 & 26,2 & 5,2 & 5,4 \\
\hline
\end{tabular}

\section{Prueba de normalidad}

Tabla 3

Prueba de normalidad Shapiro-Wilk

\begin{tabular}{lccc}
\hline Grupos & \multicolumn{3}{c}{ Shapiro-Wilk } \\
\cline { 2 - 4 } & Estadístico & gl & Sig. \\
\hline Grupo A sin aletas & 902 & 15 &, 103 \\
\hline Grupo B sin aletas &, 898 & 15 &, 089 \\
\hline Grupo C sin aletas &, 808 & 15 &, 005 \\
\hline Grupo A con aletas &, 964 & 35 &, 296 \\
\hline Grupo B con aletas &, 975 & 10 &, 934
\end{tabular}

Pruebade muestras relacionadas (pruebat paragrupos con distribución normal)

Tabla 4

Prueba t entre los diferentes grupo

\begin{tabular}{|c|c|c|c|c|c|c|c|c|}
\hline \multirow{3}{*}{ Pares de grupos } & \multicolumn{5}{|c|}{ Diferencias relacionadas } & \multirow{3}{*}{$\mathrm{t}$} & \multirow{3}{*}{ Gl } & \multirow{3}{*}{$\begin{array}{l}\text { Sig. } \\
\text { (bilat } \\
\text { eral) }\end{array}$} \\
\hline & \multirow[t]{2}{*}{ Media } & \multirow[t]{2}{*}{$\begin{array}{l}\text { Desv. } \\
\text { típica }\end{array}$} & \multirow[t]{2}{*}{$\begin{array}{l}\text { Error } \\
\text { tip, de } \\
\text { media }\end{array}$} & \multicolumn{2}{|c|}{$\begin{array}{c}95 \% \text { de intervalo } \\
\text { de confianza para } \\
\text { la diferencia } \\
\end{array}$} & & & \\
\hline & & & & Inferior & Superior & & & \\
\hline $\begin{array}{c}\text { Grupo A sin aletas } \\
\text { Vs } \\
\text { Grupo B sin aletas }\end{array}$ & $-13,86$ & 7,855 & 2,028 & $-18,216$ & $-9,517$ & $-6,837$ & 14 & 0,000 \\
\hline $\begin{array}{c}\text { Grupo A con aletas } \\
\text { Vs } \\
\text { Grupo B con aletas } \\
\end{array}$ & $-7,60$ & 9,620 & 2,484 & $-12,927$ & $-2,273$ & $-3,060$ & 14 & 0,008 \\
\hline $\begin{array}{c}\text { Grupo A sin aletas } \\
\text { Vs } \\
\text { Grupo A con aletas }\end{array}$ & 0,20 & 5,797 & 1,497 & $-3,010$ & 3,410 & 0,134 & 14 & 0,896 \\
\hline $\begin{array}{c}\text { Grupo B sin aletas } \\
\text { Vs }\end{array}$ & 6,46 & 14,297 & 3,692 & $-1,451$ & 14,384 & 1,752 & 14 & 0,102 \\
\hline
\end{tabular}

Estadísticos de contraste Wilcoxon (paragrupos con distribución no normal)

\begin{tabular}{|c|c|c|}
\hline Pares de grupos & $\mathbf{Z}$ & Sig. asintót. (bilateral) \\
\hline Grupo A sin aletas & & \\
\hline $\begin{array}{c}\text { Vs } \\
\text { Grupo C sin aletas }\end{array}$ & $-3,408^{\mathrm{a}}$ & 0,001 \\
\hline Grupo B sin aletas & & \\
\hline $\begin{array}{c}\text { Vs } \\
\text { Grupo C sin aletas }\end{array}$ & $-3,411^{a}$ & 0,001 \\
\hline
\end{tabular}

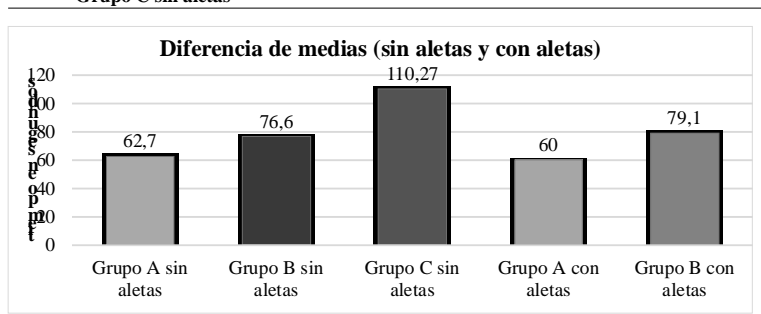

Figura 3.. Diferencia de medias de los 3 niveles sin aletas del test 1 y los 2 niveles con aleas del test 2

\section{Discusión}

Como puede observarse, las aletas mejoran de forma significativa el tiempo de ejecución del rescate acuático planteado en este estudio. Los resultados coinciden con los de otros trabajos previos $(16,17)$.Además, el empleo de las aletas hace que el 77,7\% de la muestra (35 de 45) pase a formar parte del grupo de nivel alto ( $<70$ segundos en el test) y obtenga resultados estadísticamente similares, a los obtenidos por los aspirantes más rápidos en el test 1 (sin aletas). El 22,3\% de la muestra (10 de 45) obtuvo tiempos estadísticamente similares al grupo de nivel medio. Dicho de otro modo, la utilización de las aletas permite que el $77,7 \%$ de los aspirantes a socorrista, obtengan marcas de tiempo que sólo están al alcance del 33\% de la muestra, cuando el test se realiza sin ayuda de las aletas.

La desviación típica de los tiempos obtenidos en el test, es menor cuando la muestra utiliza aletas (9,57 segundos) que cuando no las emplea (25,34 segundos). Este resultado confirma lo comunicado por otros autores (16). Por lo tanto, el empleo de las aletas, provoca que el nivel de los socorristas sea más parecido entre ellos.

Los resultados de esta investigación demuestran que cuando los participantes realizan la prueba sin aletas, se forman tres grupos de 15 sujetos cada uno, con tres niveles de destreza en el agua estadísticamente diferentes entre ellos (nivel alto, nivel medio y nivel bajo):

- El grupo A(nivel alto sin aletas), es estadísticamente diferente al Grupo B (nivel medio) sin aletas ( $\mathrm{p}<0.001$ ).

- El Grupo A (nivel alto sin aletas), es estadísticamente diferente al Grupo C (nivel bajo) sin aletas ( $\mathrm{p}<0,001$ ).

- El Grupo B (nivel medio) sin aletas, es estadísticamente diferente al Grupo C (nivel bajo) sin aletas $(\mathrm{p}<0,001)$.

En cambio, cuando esta misma población realiza el test 2 (con aletas), la muestra sólo puede ser dividida en dos grupos con niveles de destreza en el agua, estadísticamente diferentes (nivel alto y nivel medio):

- El Grupo A(nivel alto) con aletas, es estadísticamente diferente al Grupo B (nivel medio) con aletas ( $p<0,008$ ).

Es muy importante destacar que en el test 2 (con aletas), ningún participante ha superado el límite de 87 segundos, que le haría formar parte del grupo de nivel bajo. Por lo tanto, las aletas mejoran significativamente el nivel de destreza de sujetos con nivel más bajo. En cambio, aquellos aspirantes a socorristas que fueron incluidos en el grupo de nivel más alto al hacer el test 1 (sin aletas), no son estadísticamente diferentes a los que obtienen mejores marcas al realizan el test 2 (con aletas).

La considerable mejora del rendimiento de los participantes con menor nivel de destreza en el agua al utilizar las aletas, podría deberse a que cuando no se dispone de aletas, los socorristas suelen utilizar gestos técnicos iguales o similares a la patada de braza para remolcar a las víctimas hasta la orilla. No obstante, la ejecución de esos gestos técnicos, no es ni sencilla ni intuitiva, por lo tanto, los socorristas con menor dominio de esta destreza, ven incrementado desproporcionalmente el tiempo del rescate acuático. En cambio, la patada de crol de espalda que se emplea con las aletas en los remolques de accidentados, es muy propulsiva y sencilla de ejecutar, incluso para aquellos sujetos que no disponen de un buen nivel de destreza en el agua.

Por otro lado, aquellos participantes que sin ayuda de las aletas obtuvieron buenos resultados, no se han visto tan favorecidos por el uso de este material. No obstante, eso no significa que las aletas no sean útiles o recomendables para los socorristas más hábiles. De hecho, se sabe que en rescates largos, con víctimas corpulentas o con un entorno cambiante (playas), las aletas pueden determinar el éxito o el fracaso de la intervención(20).

Estos resultados tienen una enorme relevancia práctica, ya que en la actualidad, existen grandes diferencias de nivel de destreza entre los socorristas titulados. Posiblemente, porque las diferentes normativas que regulan los niveles de exigencia necesarios para ser socorrista, adolecen de una sistemática falta de homogeneidad. Sirva de ejemplo que en algunas Comunidades Autónomas de España, ni siquiera incluyen prue- 
bas de aptitud física, en las que sea necesario utilizar las aletas o exigen diferentes baremos de tiempo para las mismas pruebas.

En definitiva, los resultados obtenidos en este estudio, demuestran que en el grupo estudiado, las aletas son un material de gran utilidad en el ámbito del salvamento y socorrismo. Por este motivo, sería recomendable la inclusión de criterios de evaluación relacionados con el manejo de las aletas, en todos los cursos de formación de socorrismo. En esta misma línea, resulta paradójico que a pesar de que se ha demostrado que las aletas son un material eficaz en los rescates acuáticos, las Administraciones Sanitarias no consideran necesario u obligatorio, que los socorristas dispongan de aletas en los espacios acuáticos de grandes dimensiones. Esta situación resulta todavía más inexplicable, cuando es sabido que el coste de adquisición de las aletas es muy bajo.

\section{Conclusiones}

Finalmente, se pueden obtener las siguientes conclusiones en el grupo estudiado:

a) En general, la utilización de las aletas disminuye el tiempo empleado en el rescate de forma significativa (18,8 segundos). Este es un factor determinante para las personas que se encuentran en parada cardiorespiratoria.

b) Las aletas son especialmente adecuadas para los socorristas con peor nivel de destreza en el agua.

c) Aunque las aletas son recomendables para todos los socorristas, las marcas de los socorristas con mayor nivel de destreza, no mejoró de forma significativa.

d) El empleo de aletas hace que los niveles de los socorristas sea más parecido.

Agradecimiento: Todos los datos han sido cedidos por la Federación Madrileña de Salvamento y Socorrismo a la que agradecemos su colaboración

\section{Referencias}

1. Szpilman D, Webberemail J, Quanemail L, Bierensemail J, Morizot-Leiteemail L, Langendorferemail SJ, et al. Creating a drowning chain of survival. Resuscitation. 2014;85(9):1149 - 52.

2. World Health Organization. Global report on drowning: preventing a leading killer. In: Organization WH, editor. Geneva, Switzerland: WHO Press, World Health Organization; 2014.

3. Pino Espinosa J.Jornadas Técnicas de Salvamento y Socorrismo 2015. In: Socorrismo FES, editor. Informe Nacional de Ahogamientos; Madrid2015

4. Real Federación Española de Salvamento y Socorrismo (RFESS). Informe nacional de ahogamientos producidos del 1 de enero al 31 de diciembre de 2015. Madrid: Real Federación Española de Salvamento y Socorrismo (RFESS); 2015.

5. Abraldes JA, Rubio JA. Estudio de los Recursos Materiales de intervención en las playas de la Región de Murcia. Actas del Congreso del III Congreso de la Asociación Española de Ciencias del Deporte. Valencia: Universidad de Valencia; 2004.

6. Palacios Aguilar J. Salvamento Acuático: un estudio de la realidad del salvamento y socorrismo en las playas de Galicia con Bandera Azul 1996-1997. Tesis doctoral. A Coruña: Universidade Da Coruña; 1998.

7. Pascual LM, Sanz P, Barrio B. Campaña piscina azul 2000 Libro de actas del $2^{\circ}$ Congreso de Salvamento y Socorrismo de Galicia. A Coruña: Xañino; 2001. p. 139-63.

8. Pelletier R, Gilchrist J. Fatalities in swimming pools with lifeguards: USA 2000-2008. Injury Prevention. 2011;17(4):250-3.

9. Sanz Arribas I, Cid Yagüe L, Álvarez Barrio MJ, del Campo Vecino J. La seguridad en los espacios acuáticos. In: Martínez de Haro V, Muñoa Blas J, editors. Actividad Física, Salud y Calidad de Vida vol II (CD-ROM). Segovia: CV Ciencias del Deporte; 2013. p. 7-34.

10. Schwebel DC, Heater J, Holder E, Marciani F. Lifeguards: A forgotten aspect of drowning prevention. Injure \& Violence. 2010;2(1):13.

11. Andrew J. Drowning and inmersion injury. Anaesthesia \& Intensive Care Medicine. 2011;12(9):399-402.

12. Sanz Arribas I. La coordinación de socorristas en piscinas con gran superficie de lámina de agua. Revista Internacional de Medicina y Ciencias de la Actividad Física y el Deporte. 2011;11(44):650-73.

13. Palacios Aguilar J. Socorrismo acuático profesional «formación para la prevención y la intervención de accidentes en el medio acuático». ACoruña SADEGA; 2008.

14. Sanz Arribas I. Jornadas Técnicas de Salvamento y Socorrismo 2015. In: Socorrismo FES, editor. El aro salvavidas versus el tubo de rescate en las instalaciones acuáticas; Madrid2015.

15. García Sanz A, García Sanz JL, Díez Herrero JM. Técnicas de rescate y lesión medular en el medio acuático. Real Federación Española de Salvamento y Socorrismo ed. España: Difusión Avances de Enfermería; 2015.

16. Palacios Aguilar J. II CONGRESO INTERNACIONAL DE ACTIVIDADESACUÁTICAS, SALVAMENTOY SOCORRISMO «La importancia de la capacitación en las actividades acuáticas, socorrismo y el salvamento». Técnicas para incorporar en el salvamento: El beneficio en la utilización de aletas en socorrismo acuático; 12, 13 y 14 de octubre; POSADAS. Misiones. Argentina2012.

17. Abraldes JA, Soares S, Lima AB, Fernandes R, Vilas-Boas JP. The Effect of Fin Use on the Speed of Lifesaving Rescues. International Journal of Aquatic Research \& Education. 2007;1(4):329-40.

18. Abraldes JA, Fernandes RJ, Soares S, Lima.; AB, Vilas-Boas JP. Assessment of A Lifesaver's Instantaneous Velocity in Mannequin Carry using Diferent Types of Fins. The Open Sports Sciences Journal,. 2010;3(19):19-21.

19. Odriozola Sánchez J. Manual del Socorrista en Playa, embarcación y moto acuática. Cantabria: Federación Cántabra de Salvamento y Socorrismo; 2011.

20. Palacios Aguilar J. IV Congreso Internacional de Salvamento Acuático, Rescate y Reanimación Cardiopulmonar «El Socorrismo en la actualidad, una actividad vital y cada vez más compleja»»». El beneficio de la utilización de aletas en Socorrismo Acuático; 24, 25 y 26 de Septiembre de 2010; POSADAS. Misiones. Argentina: UAI UniversidadAbierta Interamericana; 2010.

21. Abelairas-Gomez C, Barcala-Furelos R, García-Soidan JL, López-García S, Romo-Pérez V. The use of automatic compresion device on the cardiopulmonary resucitation by lifeguards. Motricidad European Journal of Human Movement. 2012;29:17-28.

22. Szpilman D, Marcio S. In-water resuscitation-is it wortwhile? Resuscitation. 2004;63(1):25-31.

23. Neumar RWC, Shuster M, Callaway CW, Gent LM, Atkins DL, Bhanji F, et al. American Heart Association Guidelines Update for Cardiopulmonary Resuscitation and Emergency Cardiovascular Care. Supplement to Circulation. 2015;132(8):S315-S67.

24. Abelairas Gómez C, Romo Pérez V, Barcala Furelos R. Efecto de la fatiga física del socorrista en los primeros cuatro minutos de la reanimación cardiopulmonar posrescate acuático. Emergencias. 2013;25:184-90.

25. Ellis \& Associates. El profesional del rescate acuático. Barcelona: Paidotribo; 2002.

26. Marek Rejman M, Wiesner W, Silakiewicz P, Klarowicz A, Abraldes JA. Comparison of temporal parameters of swimming rescue elements when performed using dolphin and flutter kick with fins didactical approach. Journal of Sports Science and Medicine. 2012;11:682-9.

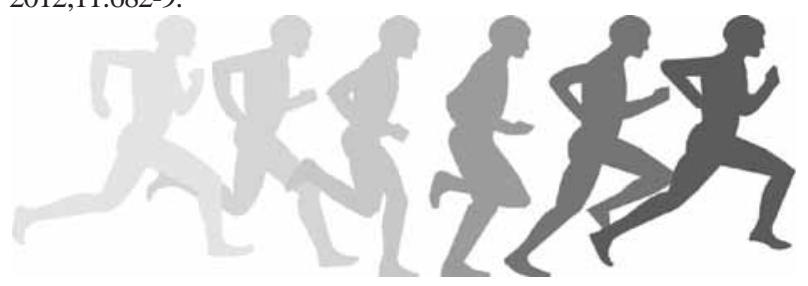

